Method 44 children with AIH (33 AIH-1 and 11 AIH-2, median age 13.5 yrs, 23 females), 65 FDR from 34 families [23 fathers, 47 yrs (38-58); 28 mothers, 44 yrs (24-53) and 14 siblings, 7 females, 13 yrs (5-24)] and 42 healthy subjects [HS, 36 yrs (22-54), 37 females] were studied. Tregs were purified from PBMCs using immunomagnetic beads and their phenotype and frequency was assessed by flowcytometry. CD2 $5^{\text {neg }}$ cells were used as responders in co-culture with Tregs and their proliferation was measured by ${ }^{3} \mathrm{H}$ thymidine incorporation. HLA genotyping was performed by PCR using gene specific primers.

Results The frequency of the disease predisposing gene HLA DR3 was significantly higher in patients $(71 \%)$ and their FDR $(56 \%)$ than in HS $(23 \%, p<0.0001$ and $p<0.005)$. The frequency of homozygous DR3 was higher in patients $(29 \%)$ than in FDR $(9 \%, p=0.015)$ and HS $(0 \%, p=0.001)$. In patients the frequency of HLA A1-B8-DR3 haplotype $(42 \%)$ was higher than in FDR $(27 \%, p=0.15)$ and HS $(16 \%, \mathrm{p}=0.02)$. The frequency of conventional CD $4{ }^{\text {pos }} \mathrm{CD} 25^{\mathrm{pos}}$ Tregs was lower in patients $(6.2 \% \pm 0.5)$ than in FDR $(9.3 \% \pm 0.7$, $\mathrm{p}=0.0016)$ and HS $(9.7 \% \pm 0.8, \mathrm{p}=0.001)$. Though the frequency of $\mathrm{CD} 4{ }^{\text {pos }} \mathrm{CD} 25^{\text {high }} \mathrm{CD} 127^{\text {neg }}$ 'True' Tregs was similar among patients, FDR and HS $(6.0 \% \pm 0.6,6.3 \% \pm 0.4$ and $6.2 \% \pm 0.5)$, their suppressive function was lower in patients $\left(13.6 \%\right.$ reduction of CD25 ${ }^{\text {neg }}$ cell proliferation) than in $\operatorname{FDR}(28.8 \%, \mathrm{p}=0.007)$ and $\mathrm{HS}(36.9 \%$, $\mathrm{p}<0.0001)$. Among subjects positive for HLA DR3, the frequency of conventional Tregs was lower in patients $(5.6 \% \pm 0.5)$ than in FDR (8.4\% $\pm 0.97, p=0.01)$ and in HS $(9.1 \% \pm 1.4, p=0.006)$. Among subjects positive for HLA A1-B8-DR3 haplotype, the frequency of Tregs was lower in patients $(6.0 \% \pm 0.7)$ than in FDR $(9.1 \% \pm 1.5$, $\mathrm{p}=0.045)$ and HS $(9.8 \% \pm 1.8, \mathrm{p}=0.02)$.

Conclusion A numerical and functional impairment of Tregs in $\mathrm{AIH}$ patients is associated with possession of HLA disease predisposing genes, and in particular HLA DR3 homozygosity. Possession of DR3 was not associated to a similar immune regulatory impairment in FDR and HS, suggesting that a gene dose effect contributes to the impairment of immunoregulation and to the development of AIH.

\section{P05 COMBINATION OF SERUM BIOMARKERS FOR THE DIAGNOSIS OF HEPATOCELLULAR CARCINOMA}

doi:10.1136/gutjnl-2011-300857a.5

${ }^{1} \mathrm{P}$ Johnson, ${ }^{1} \mathrm{M}$ Teng, ${ }^{1} \mathrm{~S}$ Pirrie, ${ }^{2} \mathrm{D}$ Palmer, ${ }^{1} \mathrm{~S}$ Berhane, ${ }^{1} \mathrm{D}$ Stocken. ${ }^{1}$ University of Birmingham, UK; ${ }^{2}$ University of Liverpool, UK

Introduction The most commonly used serum biomarker for the diagnosis of hepatocellular carcinoma (HCC) is $\alpha$ fetoprotein (AFP). However, AFP is also modestly raised in patients with chronic liver disease, particularly in those who are at a higher risk of developing HCC. A number of other circulating markers such as des- $\gamma$ carboxy prothrombin (DCP) and the Lens culinaris agglutinin-reactive fraction of $\alpha$-fetoprotein (L3) have been shown to have diagnostic discrimination which may, in certain situations, be superior to AFP. Aim We therefore aimed to build a model that combined these biomarkers with baseline liver function tests, in order to identify those factors which predict HCC and might improve diagnostic efficacy of AFP alone.

Method A prospective study was specifically designed to assess the diagnostic efficacy of biomarkers. 461 serum samples were collected between 2007 and 2011: 252 were controls samples from patients with chronic liver disease with no evidence of HCC within 6 months of the sample; 59 were from patients with early HCC which was potentially curable and 150 were from patients with late HCC. AFP, AFP-L3 and DCP were measured in serum samples using a new micro total analysis system (Wako Chemicals GmbH, Neuss, Germany). Patients were classified into two groups (HCC, control) for the primary analysis. Independent predictors of HCC were identified from multivariable logistic regression analyses. The impact of AFP, DCP and L3 are reported based on the area under the receiver operator curve (AUC).

Results Univariate analysis showed AFP alone to have $\mathrm{OR}=2.96$ (2.34 to $3.7395 \% \mathrm{CI}$ ), AUC $=0.8723, \mathrm{p}<0.001$. A multivariable model selected age, sex, AST, ALP and INR as independent predictors of HCC with an overall model AUC $=0.8857$. Including AFP, DCP and L3 to this model increased the AUC to 0.9489, 0.9290, 0.9254 respectively. The final model included age, sex, AFP, DCP and INR gave an AUC of 0.9661 (95\% CI 0.95 to 0.98 ) for all patients and 0.944 (95\% CI 0.914 to 0.974 ) for early HCC patients.

Conclusion A combination of serum markers may produce a clinically useful diagnostic test for HCC compared to AFP alone. Further validation of our model is underway on an independent dataset. These findings suggest that our model may enhance or replace the current screening approach for patients with liver cirrhosis, particularly since it appears to have efficacy even in patients with early, potentially curable, disease.

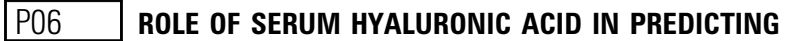 MORTALITY IN PATIENTS WITH CHRONIC LIVER DISEASE}

doi:10.1136/gutjnl-2011-300857a.6

${ }^{1} \mathrm{~N}$ Plevris, ${ }^{2} \mathrm{~N}$ C Mcavoy, ${ }^{2} \mathrm{P}$ C Hayes. ' University of Edinburgh, UK; ${ }^{2}$ Department of Hepatology, Royal Infirmary of Edinburgh, UK

Introduction Currently liver biopsies are used to assess extent of liver fibrosis and provide prognostic information in liver disease. Hyaluronic acid (HA) is a non-invasive serum marker that has been shown to correlate well with different degrees of hepatic fibrosis. Despite this the prognostic abilities of HA still remain to be established.

Aim In this study we aim to establish the relationship between levels of serum HA and survival, as well as determine the sensitivity and specificity of HA for predicting death in patients with chronic liver disease of varying aetiology.

Method Patients seen at the department of hepatology, Royal Infirmary of Edinburgh between 1995 and 2010 who had serum HA levels measured were followed up for death from the date of HA measurement until the 1 November 2010 by examination of clinical records on TRAK (NHS Lothian). The cumulative probability for survival from liver related death and overall deaths at 1-, 5- and 8-year follow-up was determined. Receiver operating characteristic (ROC) curves were generated to assess the sensitivity and specificity of HA levels at predicting liver-related death at 1, 3 and 5 years.

Results HA levels were available for 632 patients. The median follow-up time (from HA measurement to death or 'last known alive') was 2.7 years (range $0.0-8.0$; IOR 0.9-4.4). Survival analysis showed that HA levels $>400 \mu \mathrm{g} / \mathrm{l}$ are associated with a significantly lower probability of survival from liver related death compared to patients with values of $<100 \mu \mathrm{g} / 1$ and $100-400 \mu \mathrm{g} / \mathrm{l}$ at 1 year $(88 \%$, $\mathrm{p}<0.01), 5$ years $(68 \%, \mathrm{p}<0.001)$ and 8 years $(49 \%, \mathrm{p}<0.05)$. In addition, the probability of survival from liver related death at 8 years was also significantly less in patients with HA value of $100-400 \mu \mathrm{g} / 1$ compared to patients with HA $<100 \mu \mathrm{g} / 1$ (83\%, $p<0.05)$. Similar results were observed when assessing probability of survival from overall deaths. The unadjusted area under the ROC curve for predicting liver related mortality for $\mathrm{HA}$ at 1-, 3- and 5 years was 0.89 (CI 95\% 0.84 to 0.94 ), 0.85 (CI 95\% 0.79 to 0.91 ) and 0.79 (CI $95 \% 0.71$ to 0.87 ) respectively. Optimum cut-off value for 1-, 3-and 5 year for predicting liver death was $326 \mu \mathrm{g} / 1$ (88\% sensitivity/79\% specificity), $267 \mu \mathrm{g} / 1$ (74\% sensitivity/79\% specificity) and $203 \mu \mathrm{g} / 1$ (71\% sensitivity/72\% specificity) respectively.

Conclusion In this study we have shown that patients with high levels of serum HA have an increased liver and all cause mortality. In addition, HA levels are both sensitive as well as specific at determining liver related mortality. This highlights the potential use of 